\title{
Arcsine Laws in Stochastic Thermodynamics
}

\author{
Andre C. Barato, ${ }^{1, *}$ Édgar Roldán, ${ }^{1,2, \dagger}$ Ignacio A. Martínez, ${ }^{3}$ and Simone Pigolotti ${ }^{4}$ \\ ${ }^{1}$ Max Planck Institute for the Physics of Complex Systems, Nöthnizer Strasse 38, 01187 Dresden, Germany \\ ${ }^{2}$ Abdus Salam International Centre for Theoretical Physics, Strada Costiera 11, 34151, Trieste, Italy \\ ${ }^{3}$ Departamento de Estructura de la Materia, Física Termica y Electronica and GISC, \\ Universidad Complutense de Madrid 28040 Madrid, Spain \\ ${ }^{4}$ Okinawa Institute of Science and Technology Graduate University, Onna, Okinawa 904-0495, Japan
}

(Received 1 March 2018; published 28 August 2018)

\begin{abstract}
We show that the fraction of time that a thermodynamic current spends above its average value follows the arcsine law, a prominent result obtained by Lévy for Brownian motion. Stochastic currents with long streaks above or below their average are much more likely than those that spend similar fractions of time above and below their average. Our result is confirmed with experimental data from a Brownian Carnot engine. We also conjecture that two other random times associated with currents obey the arcsine law: the time a current reaches its maximum value and the last time a current crosses its average value. These results apply to, inter alia, molecular motors, quantum dots, and colloidal systems.
\end{abstract}

DOI: 10.1103/PhysRevLett.121.090601

In 1940, Paul Lévy calculated the distribution of the fraction of time $\mathcal{T}$ that a trajectory of Brownian motion stays above zero [1]. Lévy proved that this fraction of time is distributed according to

$$
P(\mathcal{T})=\frac{1}{\pi} \frac{1}{\sqrt{\mathcal{T}(1-\mathcal{T})}} .
$$

This result and related extensions are often referred to as the "arcsine law" [2-5]. The name stems from the fact that the cumulative distribution of $\mathcal{T}$ reads $F(\mathcal{T})=$ $\int_{0}^{\mathcal{T}} P\left(\mathcal{T}^{\prime}\right) d \mathcal{T}^{\prime}=(2 / \pi) \arcsin (\sqrt{\mathcal{T}})$. A counterintuitive aspect of the $U$-shaped distribution (1) is that its average value $\langle\mathcal{T}\rangle=1 / 2$ corresponds to the minimum of the distribution, i.e., the less probable outcome, whereas values close to the extrema $\mathcal{T}=0$ and $\mathcal{T}=1$ are much more likely. Brownian trajectories with a long "winning" (positive) or "losing" (negative) streak are quite likely.

Several phenomena in physics and biology have been shown to be described by the arcsine law and related distributions. Examples include conductance in disordered materials [6,7], chaotic dynamical systems [8], partial melting of polymers [9], quantum chaotic scattering [10], and generalized fractional Brownian processes [11]. Notably, the arcsine law (1) has also been explored in finance [12], where investment strategies can lead to a much smaller alternance of periods of gain and loss than one would expect based on naive arguments.

Recent theory and experiments extended thermodynamics to mesoscopic systems that are driven away from equilibrium [13-19]. Mesoscopic systems operate at energies comparable with the thermal energy $k_{B} T$, where $k_{B}$ is the Boltzmann constant and $T$ is the temperature. At these energy scales, observables such as work, heat, entropy production, and other thermodynamic currents are not deterministic as in macroscopic thermodynamics, but rather stochastic quantities [20].

While the concept of a fluctuating entropy was already suggested by the forefathers of thermodynamics and statistical physics [21], the universal statistical properties of thermodynamic currents discovered in the last two decades have extended thermodynamics, providing novel insights that also apply to the nanoscale. Prominent examples are fluctuation relations [22-28], which generalize the second law of thermodynamics. More recently, several other universal results have been obtained. They include a relation between precision and dissipation known as the thermodynamic uncertainty relation [29-31], stopping-time and extreme-value distributions of entropy production (and related observables) [32-35], and efficiency statistics for mesoscopic machines [36-39].

In this Letter, we find a new universal result about the statistics of thermodynamic currents. We demonstrate that the fraction of time $\mathcal{T}_{1}$ that a generic thermodynamic current stays above its average value (see Fig. 1) is distributed according to Eq. (1). This result is valid for mesoscopic systems in a nonequilibrium steady state and also for periodically driven mesoscopic systems. The proof of the arcsine law for $\mathcal{T}_{1}$ is based on a theorem for Markov processes that has hitherto remained unexplored in physics [40]. Our results are verified with experimental data from a Brownian Carnot engine [39]. Based on numerical evidence, we also conjecture that two other random variables related to thermodynamic currents are distributed according to (1): the last time a fluctuating current crosses its average $\mathcal{T}_{2}$ and the time elapsed until a current reaches its maximal 


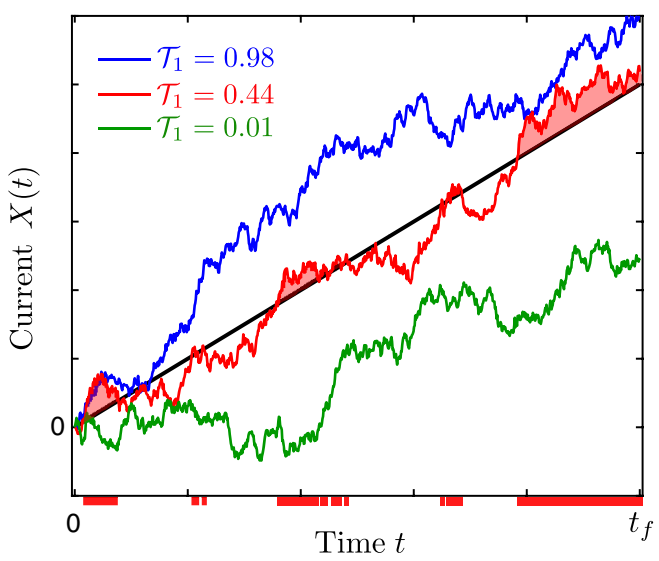

FIG. 1. Illustration of the fraction of time $\mathcal{T}_{1}$ elapsed by a thermodynamic current above its average. The figure shows three different realizations of a stochastic current (colored lines) and its average linear growth (black line). For the example of the red trajectory, we mark in the $x$ axis the time intervals for which the trajectory stays above the average (red shaded areas). The values of $\mathcal{T}_{1}$ for the three trajectories are shown in the legend.

deviation from the average $\mathcal{T}_{3}$. These results are valid in the limit of large observation times.

Arcsine law for $\mathcal{T}_{1}$. - We consider small nonequilibrium physical systems in contact with one or several thermal and/ or particle reservoirs at thermal equilibrium, for instance, a single enzyme (the system) immersed in a solution (the reservoir) that contains both substrate and product molecules. The system is in a nonequilibrium steady state if the concentrations of substrate and product in the large reservoir and the rate at which the enzyme consumes the substrate are approximately constant. In this example, the chemical potential difference between substrate and product is the thermodynamic force that drives the system out of equilibrium.

A vast class of these systems in physics and biochemistry can be described by Markov processes within the framework of stochastic thermodynamics [14]. In this framework, transition rates are associated with thermodynamic quantities, such as temperature and chemical potential, through the generalized detailed balance relation [28]. As a consequence, integrated probability currents represent thermodynamic currents. At steady state, their average rate is constant, leading to a linear increase (or decrease) with time of the average thermodynamic currents. The fraction of time that a stochastic thermodynamic current $X(t)$ spends above its average value $\langle X(t)\rangle$ during an observation time $t_{f}>0$ is defined as

$$
\mathcal{T}_{1} \equiv \frac{1}{t_{f}} \int_{0}^{t_{f}} \theta(X(t)-\langle X(t)\rangle) d t
$$

where $\theta(x)$ is the Heaviside function. This random variable $\mathcal{T}_{1}$ is illustrated in Fig. 1.
Our main result is that, for any thermodynamic current in a small system at steady state that is described by a Markov process, the probability density of $\mathcal{T}_{1}$, for large $t_{f}$, is given by Eq. (1). Hence, stochastic trajectories for which currents such as heat, work, and entropy production stay all the time above or below their average value are the most likely. The striking universality of this result is illustrated in Fig. 2, where we show numerical simulations of three models of different physical systems: a double quantum dot [44], a molecular motor [45], and a driven colloidal particle [34]. The mathematical proof of this result requires the use of a theorem for Markov chains that establishes an arcsine law for a random variable different from a current [40] and a suitable mapping between two Markov chains [41]. Interestingly, the proof also extends to time-symmetric observables such as activity (or frenesy [46]) (see Supplemental Material [41] for details). A key idea behind this proof is as follows. In contrast to Brownian motion, currents in Markov processes are sums of correlated random variables. However, if we consider a counting procedure for which the current is updated only when an
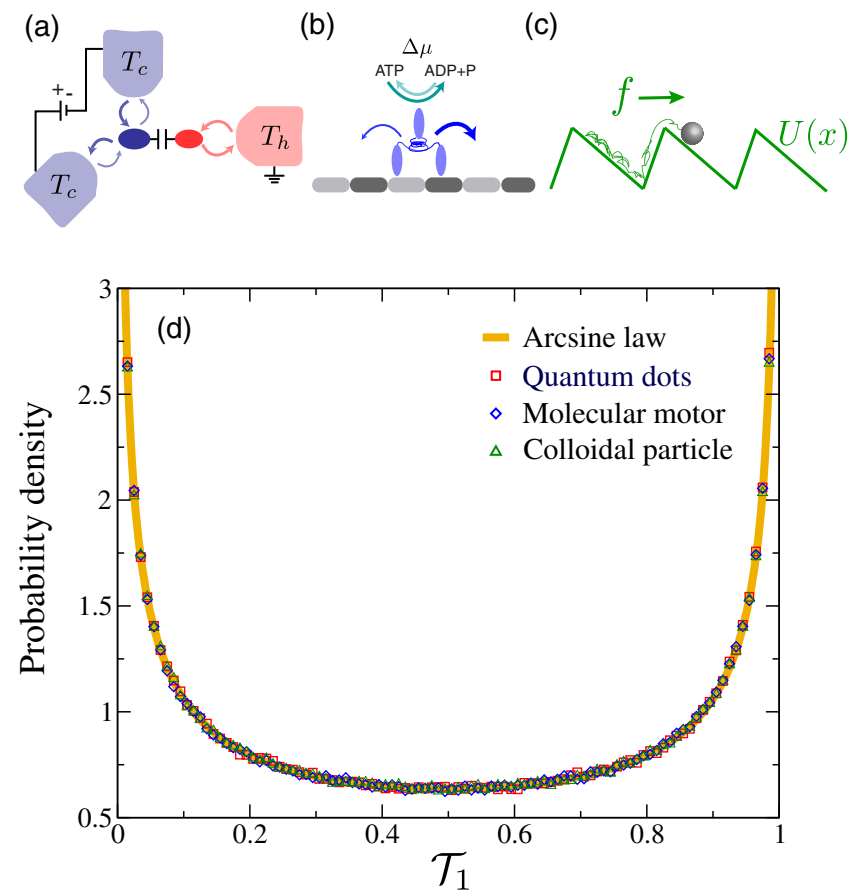

FIG. 2. Numerical illustration of the steady-state arcsine law for $\mathcal{T}_{1}$. (a)-(c) Graphical illustration of the models. (a) A double quantum dot. (b) A molecular motor. (c) A colloidal particle on a periodic potential subjected to an external force. The thermodynamic currents that we consider are the electron current through the cold dot, the net number of steps of the motor, and the net position of the particle, respectively. (d) Comparison of the prediction of Eq. (1) (orange line) with numerical simulations for the three different models. For each curve, the number of realizations is $10^{6}$ and the total time is $t_{f}=10^{4}$ (quantum dots and molecular motor) and $t_{f}=10$ (colloidal particle). Details of the three models are given in the Supplemental Material [41]. 
arbitrary reference state is revisited, the increments of the current within this procedure become independent random variables due to the so-called strong Markov property [40].

Small thermodynamic engines and several other systems of physical and technological interest are driven by an external periodic protocol $[18,47]$. Such periodically driven systems are described by the Markov process with timeperiodic transition rates. Nevertheless, in the long time limit, it is possible to describe periodically driven systems as steady states of Markov processes with time-independent transition rates $[48,49]$. Hence, the arcsine law for $\mathcal{T}_{1}$ is also valid for periodically driven systems, in the limit at which the observation time $t_{f}$ is much larger than the period of the protocol. We have illustrated this result with numerical simulations of two models: a colloidal particle in a time-periodic potential and a theoretical model for a Brownian Carnot engine [41].

Conjecture for $\mathcal{T}_{2}$ and $\mathcal{T}_{3}$.-For Brownian motion, two other random variables obey Lévy's arcsine law (1). One is the last time the walker crosses zero and the other is the time the position of the walker reaches its maximum value. The equivalent random variables for the present case are defined as follows. The fraction of time elapsed until a current crosses its average value for the last time $\mathcal{T}_{2}$ is defined as

$$
\mathcal{T}_{2} \equiv \sup _{t \in\left[0, t_{f}\right]}\left\{\frac{t}{t_{f}}: \Delta_{X}(t)=0\right\}
$$

where $\Delta_{X}(t) \equiv X(t)-\langle X(t)\rangle$. The time $t_{\text {sup }}$ is defined as the time at which $\Delta_{X}(t)$ attains its supremum, i.e., $\Delta_{X}\left(t_{\text {sup }}\right)=\sup _{t \in\left[0, t_{f}\right]} \Delta_{X}(t)$. The fraction of time elapsed until a current reaches its maximal deviation above its average value is

$$
\mathcal{T}_{3} \equiv \frac{t_{\text {sup }}}{t_{f}}
$$

We have verified numerically that both $\mathcal{T}_{2}$ and $\mathcal{T}_{3}$ tend to the distribution (1) in the limit of large $t_{f}$. Specifically, we have performed numerical simulations of the models shown in Figs. 3(a)-3(c) with a finite observation time $t_{f}$, where $t_{f}$ is small enough such that we can accurately determine the third cumulant associated with the current, which is nonzero for all models (see Supplemental Material [41]). Our simulations then probe large non-Gaussian fluctuations and, therefore, they test arcsine laws for Markov processes, beyond Brownian motion.

As shown in Fig. 3(d), we have performed a finite-size scaling analysis of the Kolmogorov-Smirnov (K-S) statistic for $\mathcal{T}_{1}, \mathcal{T}_{2}$, and $\mathcal{T}_{3}$, with respect to the arcsine distribution (1), as a function of $t_{f}$. All random variables show the same behavior: for large times, the K-S statistic goes to zero as the power law $t_{f}^{-1 / 2}$. For a finite number $n$ of independent identically distributed random variables, the K-S statistic
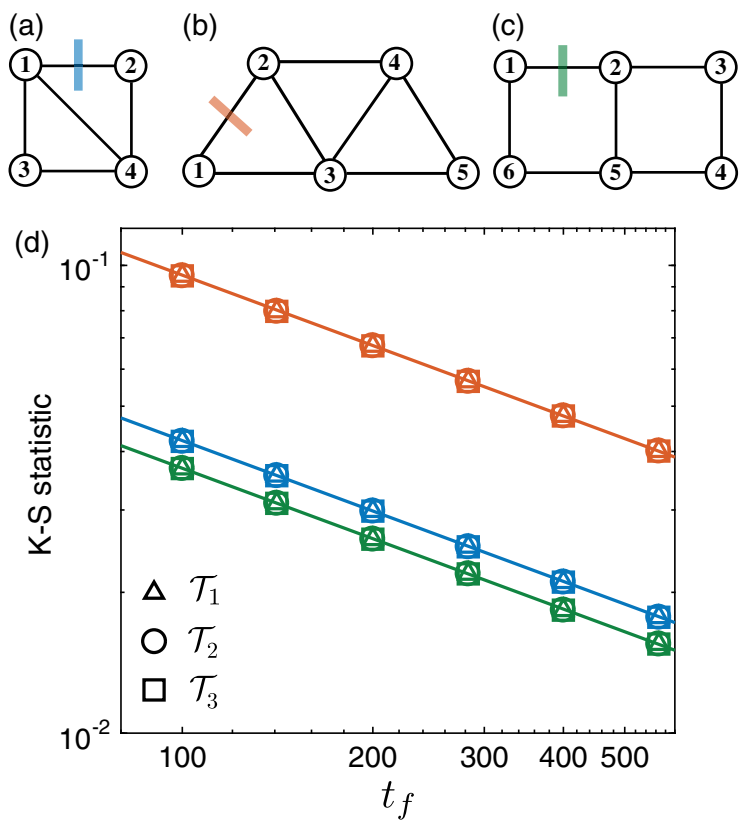

FIG. 3. Numerical verification of the three arcsine laws. (a)-(c) Graphical illustration of minimal stochastic models with four (a), five (b), and six (c) different states. Each state is represented by a circle with a number and the lines represent nonzero transition rates. For all models, we have evaluated the current from state 1 to state 2, as indicated with bars in the figures. (d) Kolmogorov-Smirnov statistic between a reference $\mathcal{T}$ described by the arcsine law (1) and $\mathcal{T}_{1}$ (triangles), $\mathcal{T}_{2}$ (circles), and $\mathcal{T}_{3}$ (squares), as a function of $t_{f}$. Different colors represent results for model (a) (blue), (b) (red), and (c) (green). Each symbol is obtained from $10^{7}$ numerical simulations of total duration $t_{f}$. The lines are guides to the eye and are given by $\sim t_{f}^{-1 / 2}$. Details of the three models are given in the Supplemental Material [41].

scales as $n^{-1 / 2}$ [50]. The scaling observed in Fig. 3(d) suggests that also the K-S statistic of $\mathcal{T}_{1}, \mathcal{T}_{2}$, and $\mathcal{T}_{3}$ follow a similar scaling, with their distribution for finite observation time $t_{f}$ approaching the distribution in Eq. (1) as $t_{f}^{-1 / 2}$. We have also performed this analysis for the models in Fig. 2 [41]. Based on this numerical evidence, we then conjecture that $\mathcal{T}_{2}$ and $\mathcal{T}_{3}$ are also distributed according to (1).

Experimental results.-Heat engines are paradigmatic examples of periodically driven systems [51]. We test the arcsine law for $\mathcal{T}_{1}$ using experimental data of a Brownian Carnot engine [39]. The working substance of the engine is a single optically trapped colloidal particle of radius $\mathrm{R}=$ $500 \mathrm{~nm}$ immersed in water. The particle is trapped in a time-periodic harmonic potential $U(x, t)=\kappa(t) x^{2}(t) / 2$, whose stiffness $\kappa(t)$ is externally controlled along a period $\tau$ between the minimum value $\kappa_{\mathrm{I}}=\kappa(0)=$ $(2.0 \pm 0.2) \mathrm{pN} \mu \mathrm{m}^{-1}$ and the maximum value $\kappa_{\mathrm{III}}=\kappa(\tau / 2)=$ $(20.0 \pm 0.2) \mathrm{pN} \mu \mathrm{m}^{-1}$. In addition, the effective kinetic temperature of the particle is switched periodically between a cold $T_{\mathrm{c}}=300 \mathrm{~K}$ and a hot temperature $T_{\mathrm{h}}=526 \mathrm{~K}$. The effective kinetic temperature is controlled with an external 


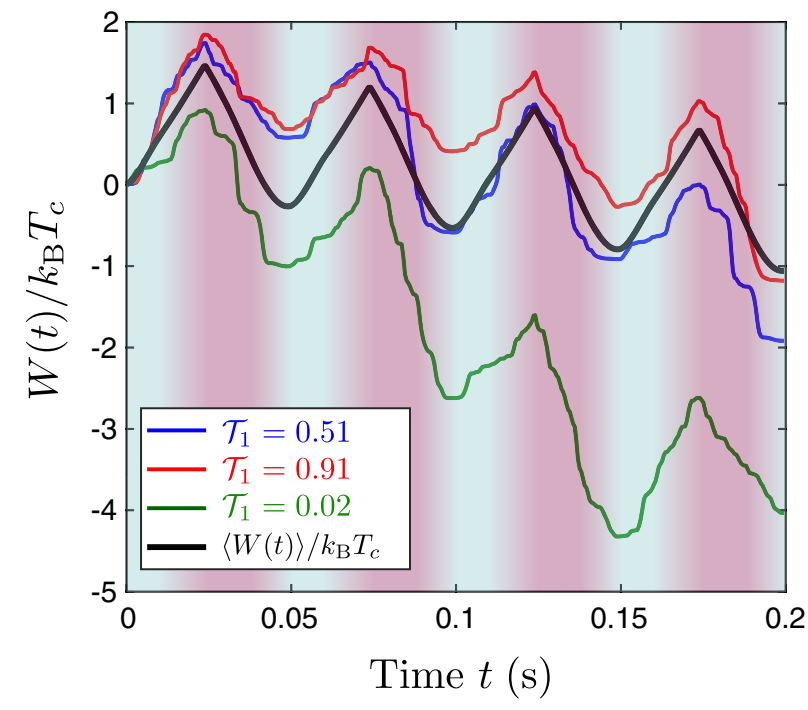

FIG. 4. Fluctuations of $\mathcal{T}_{1}$ in the Brownian Carnot engine. Sample traces of the stochastic work exerted on the colloidal particle as a function of time. The legend indicates the corresponding value of the time $\mathcal{T}_{1}$ elapsed for each trajectory above the average value of the work (black curve). The background color illustrates the effective kinetic temperature of the particle during the operation of the engine, with $T_{c}=300 \mathrm{~K}$ and $T_{h}=$ $526 \mathrm{~K}$ corresponding to the minimum (blue) and maximum (red) temperatures of the engine. The isothermal steps are connected by microadiabatic protocols in which the temperature of the particle changes smoothly with time $[41,53]$.

noisy electrostatic field using the "white noise technique" [52]. The fine and simultaneous electronic control of the trap strength and the temperature of the particle allows us to implement protocols of different cycle times $\tau$ without loss of resolution, which range from $\tau=10$ to $\tau=200 \mathrm{~ms}$. The total experimental time is $50 \mathrm{~s}$ for all the values of $\tau$ [41].

A key thermodynamic current that characterizes the performance of the Brownian Carnot engine is the stochastic work $W(t)$, where we adopt the convention that negative $W(t)$ means extracted work. The stochastic work is the change of $U[x(t)]$ due to the external control exerted on the particle that leads to a time-varying stiffness $\kappa(t)$ [see Eq. (17) in [41] ]. We measure the work from experimental traces of the particle position by means of the expression $W(t)=\int_{0}^{t}\left(\partial U / \partial t^{\prime}\right) d t^{\prime}=(1 / 2) \int_{0}^{t} x^{2}\left(t^{\prime}\right) d \kappa\left(t^{\prime}\right)$. We point out that, in the asymptotic limit of large observation time $t_{f}$, the stochastic work can be approximated as an integrated probability current due to the first law. In this limit, the work $W(t) \simeq-Q_{h}(t)-Q_{c}(t)$ equals the heat taken from the hot reservoir minus the heat that flows to the cold reservoir. The contribution to the first law due to the energy change, which is not extensive in $t$, becomes negligible for large $t_{f}$ in comparison to the work and the heat exchanges.

In order to test the arcsine law, we measure the fluctuations of the fraction of time $\mathcal{T}_{1}$ that the stochastic work $W(t)$ elapses above its average value; see Fig. 4 for an

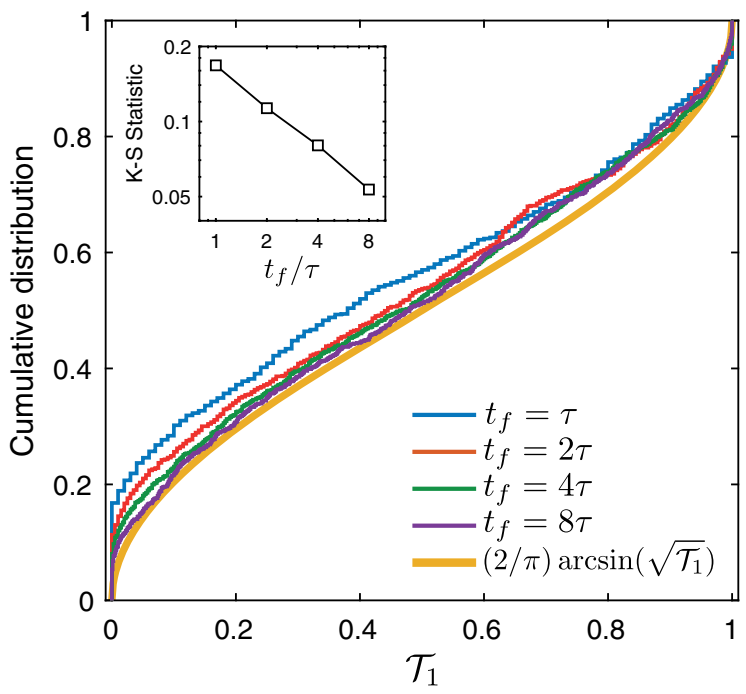

FIG. 5. Experimental verification of the arcsine law for $\mathcal{T}_{1}$ in the Brownian Carnot engine. Empirical cumulative distribution of the fraction of time $\mathcal{T}_{1}$ the work exerted on the particle elapses above its average value. The experimental data are obtained from $N=10^{3}$ cycles of the Brownian Carnot engine with cycle period $\tau=50 \mathrm{~ms}$. Different colors represent the experimental cumulative distribution of $\mathcal{T}_{1}$ calculated over different values of $t_{f}$ (see legend) and the theoretical arcsine distribution (thick orange line). (Inset) Two-sample Kolmogorov-Smirnov statistic between the empirical and theoretical cumulative distributions $[50,54]$ as a function of $t_{f}$.

illustration. We compute $\mathcal{T}_{1}=\left(1 / t_{f}\right) \int_{0}^{t_{f}} d t \theta(W(t)-\langle W(t)\rangle)$ integrating over different values of the observation time $t_{f}$, which is an integer number of periods. Since the arcsine law holds in the limit of large $t_{f}$, we perform a finite-size scaling analysis of the validity of Eq. (1). Figure 5 shows that for the experimental data the cumulative distribution of $\mathcal{T}_{1}$ converges to $(2 / \pi) \arcsin \left(\sqrt{\mathcal{T}_{1}}\right)$ when increasing the observation time $t_{f}$. We quantify the discrepancies between the experimental data and the theoretical cumulative distribution given by $(2 / \pi) \arcsin (\sqrt{\mathcal{T}})$ using the two-sample K-S statistic [50,54] (Fig. 4, inset). A finite-size scaling analysis of the K-S statistic as a function of $t_{f}$ reveals that the experimental distributions of $\mathcal{T}_{1}$ converge to the arcsine distribution. Notably, we have also demonstrated the second and third arcsine laws with the same experimental data and verified that similar results are obtained in different experimental conditions, i.e., for different values of the period $\tau$ (see Supplemental Material [41]).

Conclusion.-We have shown with theory, simulations, and experiments that the fraction of time $\mathcal{T}_{1}$ a stochastic current elapses above (or below) its average value is distributed according to Lévy's arcsine law (1) in the limit of large observation time. This result is valid for both systems in nonequilibrium steady states and for periodically driven systems such as mesoscopic engines. Based on numerical evidence, we have also conjectured that there are arcsine laws for the last time $\mathcal{T}_{2}$ at which a current crosses 
its average value and for the time $\mathcal{T}_{3}$ when a current reaches its maximal deviation from its average.

We have investigated fluctuations of mesoscopic systems described by Markovian dynamics. It is an open question whether similar results also hold for non-Markovian stochastic processes used in the description of active matter [55,56] and open quantum systems [57]. It will be interesting to investigate whether the arcsine laws for thermodynamic currents can be used to design efficient control at the nanoscale.

I. A. M. acknowledges financial support from the Spanish Government, TerMic (FIS2014-52486-R) grant and Juan de la Cierva program. We acknowledge fruitful discussions with Izaak Neri, Raphael Chetrite, and Hugo Touchette.

*barato@pks.mpg.de †edgar@ictp.it

[1] P. Lévy, Compos. Math. 7, 283 (1940); http://www.numdam .org/article/CM_1940_7_283_0.pdf.

[2] P. Erdös and M. Kac, Bull. Am. Math. Soc. 53, 1011 (1947).

[3] F. Spitzer, Trans. Am. Math. Soc. 82, 323 (1956).

[4] W. Feller, An Introduction to Probability Theory and Its Applications (Wiley, New York, 1971).

[5] S. N. Majumdar, Curr. Sci. 89, 2076 (2005).

[6] Y. V. Nazarov, Phys. Rev. Lett. 73, 134 (1994).

[7] C. W. J. Beenakker, Rev. Mod. Phys. 69, 731 (1997).

[8] T. Akimoto, J. Stat. Phys. 132, 171 (2008).

[9] G. Oshanin and S. Redner, Europhys. Lett. 85, 10008 (2009).

[10] C. Mejia-Monasterio, G. Oshanin, and G. Schehr, Phys. Rev. E 84, 035203 (2011).

[11] T. Sadhu, M. Delorme, and K. J. Wiese, Phys. Rev. Lett. 120, 040603 (2018).

[12] A. N. Shiryaev, In Mathematical Finance Bachelier Congress 2000 (Springer, New York, 2002), p. 487.

[13] C. J. Bustamante, J. Liphardt, and F. Ritort, Phys. Today 58, No. 7, 43 (2005).

[14] U. Seifert, Rep. Prog. Phys. 75, 126001 (2012).

[15] J. M. R. Parrondo, J. M. Horowitz, and T. Sagawa, Nat. Phys. 11, 131 (2015).

[16] J. P. Pekola, Nat. Phys. 11, 118 (2015).

[17] K. Proesmans, Y. Dreher, M. Gavrilov, J. Bechhoefer, and C. Van den Broeck, Phys. Rev. X 6, 041010 (2016).

[18] I. A. Martínez, É. Roldán, L. Dinis, and R. A. Rica, Soft Matter 13, 22 (2017).

[19] S. Ciliberto, Phys. Rev. X 7, 021051 (2017).

[20] K. Sekimoto, Prog. Theor. Phys. Suppl. 130, 17 (1998).

[21] J. C. Maxwell, Nature (London) 17, 278 (1878).

[22] G. N. Bochkov and Y. E. Kuzovlev, Sov. Phys. JETP 49, 543 (1979).

[23] G. Gallavotti and E. G. D. Cohen, Phys. Rev. Lett. 74, 2694 (1995).

[24] C. Jarzynski, Phys. Rev. Lett. 78, 2690 (1997).

[25] J. Kurchan, J. Phys. A 31, 3719 (1998).
[26] J. L. Lebowitz and H. Spohn, J. Stat. Phys. 95, 333 (1999).

[27] G. E. Crooks, Phys. Rev. E 60, 2721 (1999).

[28] U. Seifert, Phys. Rev. Lett. 95, 040602 (2005).

[29] A. C. Barato and U. Seifert, Phys. Rev. Lett. 114, 158101 (2015).

[30] P. Pietzonka, A. C. Barato, and U. Seifert, Phys. Rev. E 93, 052145 (2016).

[31] T. R. Gingrich, J. M. Horowitz, N. Perunov, and J. L. England, Phys. Rev. Lett. 116, 120601 (2016).

[32] K. Saito and A. Dhar, Europhys. Lett. 114, 50004 (2016).

[33] I. Neri, É. Roldán, and F. Jülicher, Phys. Rev. X 7, 011019 (2017).

[34] S. Pigolotti, I. Neri, É. Roldán, and F. Jülicher, Phys. Rev. Lett. 119, 140604 (2017).

[35] J. P. Garrahan, Phys. Rev. E 95, 032134 (2017).

[36] G. Verley, T. Willaert, C. Van den Broeck, and M. Esposito, Nat. Commun. 5, 4721 (2014).

[37] T. R. Gingrich, G. M. Rotskoff, S. Vaikuntanathan, and P. L. Geissler, New J. Phys. 16, 102003 (2014).

[38] M. Polettini, G. Verley, and M. Esposito, Phys. Rev. Lett. 114, 050601 (2015).

[39] I. A Martínez, É. Roldán, L. Dinis, D. Petrov, J. M. R. Parrondo, and R. A. Rica, Nat. Phys. 12, 67 (2016).

[40] D. A. Freedman, Proc. Am. Math. Soc. 14, 680 (1963).

[41] See Supplemental Material at http://link.aps.org/ supplemental/10.1103/PhysRevLett.121.090601 for detailed calculations, further numerical results, and further analysis of experimental data, which contains Refs. [42,43].

[42] G. L. Jones, Probab. Surv. 1, 299 (2004).

[43] K. Visscher, S. P. Gross, and S. M. Block, IEEE J. Sel. Top. Quantum Electron. 2, 1066 (1996).

[44] R. Sánchez, B. Sothmann, A. N. Jordan, and M. Büttiker, New J. Phys. 15, 125001 (2013).

[45] T. Schmiedl and U. Seifert, Europhys. Lett. 83, 30005 (2008).

[46] M. Baiesi, C. Maes, and B. Wynants, Phys. Rev. Lett. 103, 010602 (2009).

[47] S. Erbas-Cakmak, D. A. Leigh, C. T. McTernan, and A. L. Nussbaumer, Chem. Rev. 115, 10081 (2015).

[48] A. C. Barato and U. Seifert, Phys. Rev. X 6, 041053 (2016).

[49] S. Ray and A. C. Barato, Phys. Rev. E 96, 052120 (2017).

[50] A. N. Kolmogorov, G. Instit. Ital. Attuari 4, 83 (1933).

[51] S. Carnot, Réflexions sur la puissance motrice du feu et sur les machines propres à développer cette puissance (Société mathématique de France, Paris, France, 1872).

[52] I. A. Martínez, É. Roldán, J. M. R. Parrondo, and D. Petrov, Phys. Rev. E 87, 032159 (2013).

[53] I. A. Martínez, É. Roldán, L. Dinis, D. Petrov, and R. A. Rica, Phys. Rev. Lett. 114, 120601 (2015).

[54] H. Smirnov, Moscow Univ. Math. Bull. 2, 3 (1939).

[55] C. Bechinger, R. Di Leonardo, H. Löwen, C. Reichhardt, G. Volpe, and G. Volpe, Rev. Mod. Phys. 88, 045006 (2016).

[56] J. Prost, F. Jülicher, and J-F. Joanny, Nat. Phys. 11, 111 (2015).

[57] H.-P. Breuer, E.-M. Laine, J. Piilo, and B. Vacchini, Rev. Mod. Phys. 88, 021002 (2016). 